\title{
Brain metastases free survival differs between breast cancer subtypes
}

\author{
A Berghoff',2, Z Bago-Horvath', ${ }^{1,3}$, C De Vries ${ }^{1,2}$, P Dubsky ${ }^{1,4}$, U Pluschnig ${ }^{1,2}$, M Rudas ${ }^{1,3}$, A Rottenfusser ${ }^{1,5}$,

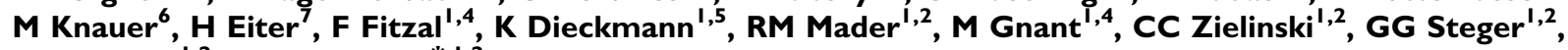 \\ M Preusser ${ }^{1,2}$ and R Bartsch*,1,2 \\ 'Comprehensive Cancer Center Vienna, Medical University of Vienna, Waehringer Guertel 18-20, A-1090 Vienna, Austria; '2Department of Medicine I, \\ Clinical Division of Oncology, Medical University of Vienna, Waehringer Guertel 18-20, A- 090 Vienna, Austria; ${ }^{3}$ Department of Pathology, Medical \\ University of Vienna, Vienna, Austria; ${ }^{4}$ Department of Surgery, Medical University of Vienna, Vienna, Austria; ${ }^{5}$ Department of Radiotherapy, Medical \\ University of Vienna, Vienna, Austria; ${ }^{6}$ Department of Surgery, Academic Teaching Hospital Feldkirch, Feldkirch, Austria; ${ }^{7}$ Department of Radiotherapy, \\ Academic Teaching Hospital Feldkirch, Feldkirch, Austria
}

BACKGROUND: Brain metastases (BM) are frequently diagnosed in patients with HER-2-positive metastatic breast cancer; in addition, an increasing incidence was reported for triple-negative tumours. We aimed to compare brain metastases free survival (BMFS) of breast cancer subtypes in patients treated between 1996 until 2010.

METHODS: Brain metastases free survival was measured as the interval from diagnosis of extracranial breast cancer metastases until diagnosis of BM. HER-2 status was analysed by immunohistochemistry and reanalysed by fluorescent in situ hybridisation if a score of $2+$ was gained. Oestrogen-receptor (ER) and progesterone-receptor (PgR) status was analysed by immunohistochemistry. Brain metastases free survival curves were estimated with the Kaplan-Meier method and compared with the log-rank test.

RESULTS: Data of 213 patients (46 luminal/I24 HER-2/43 triple-negative subtype) with BM from breast cancer were available for the analysis. Brain metastases free survival differed significantly between breast cancer subtypes. Median BMFS in triple-negative tumours was 14 months (95\% Cl: I I.34- 16.66) compared with 18 months (95\% Cl: 14.46-2I.54) in HER-2-positive tumours $(P=0.00$ I) and 34 months (95\% Cl: 23.7I -44.29) in luminal tumours $(P=0.00$ I), respectively. In HER-2-positive patients, co-positivity for ER and HER-2 prolonged BMFS (26 vs 15 m; $P=0.033$ ); in luminal tumours, co-expression of ER and PgR was not significantly associated with BMFS. Brain metastases free survival in patients with lung metastases was significantly shorter ( 17 vs 21 months; $P=0.014$ ). CONCLUSION: Brain metastases free survival in triple-negative breast cancer, as well as in HER-2-positive/ER-negative, is significantly shorter compared with HER-2/ER co-positive or luminal tumours, mirroring the aggressiveness of these breast cancer subtypes. British Journal of Cancer (2012) 1 06, 440-446. doi:I0.1038/bjc.20I I.597 www.bjcancer.com

Published online 10 January 2012

(C) 2012 Cancer Research UK

Keywords: advanced breast cancer; brain metastases; carcinomatous meningitis; human epidermal growth factor receptor 2 (HER-2)positive breast cancer; triple-negative disease

In the last decade, overall survival of metastatic breast cancer patients has improved due to advances in systemic treatment (Lin and Winer, 2007; Kiely et al, 2011). Despite this success, the rising incidence of brain metastases (BM) as late complication became a major clinical problem (Weil et al, 2005; Pestalozzi et al, 2006). About $10-15 \%$ of all metastatic breast cancer patients will eventually develop symptomatic BM during their course of disease. Brain metastases decrease quality of life and increase morbidity and mortality. Currently, survival of patients with BM ranges from 2 to 16 months (Weil et al, 2005).

Prognosis and clinical behaviour of breast cancer differs between subtypes (Perou et al, 2000; Sorlie et al, 2001; Kennecke et al, 2010). Patients with triple-negative tumours, defined by the

*Correspondence: Dr R Bartsch;

E-mail: rupert.bartsch@meduniwien.ac.at

Received 2 November 2011; revised 7 December 2011; accepted 16 December 20 I I; published online 10 January 2012 absence of oestrogen-receptor (ER), progesterone-receptor (PgR) and Her-2-receptor expression, are at higher risk of being diagnosed with BM compared with the luminal or HER-2-positive subtypes (Heitz et al, 2009). HER-2-positive patients, on the other hand, have a higher incidence of BM than patients with HER-2negative breast cancer (Sanna et al, 2007). Especially since the introduction of trastuzumab, a growing incidence of symptomatic $\mathrm{BM}$ was reported. As trastuzumab cannot penetrate trough the blood-brain barrier due to its molecular weight, a tumour cell sanctuary is created. Furthermore, trastuzumab improves systemic disease control, which leads to a 'unmasking' of BM in patients who would otherwise have died from progression of systemic disease.

Apart from triple-negative or HER-2-positive disease, established risk factors for the development of BM are young age at first diagnosis, presence of lung metastases and short disease-free interval (Weil et al, 2005).

Treatment of BM remains challenging and consists of surgery, whole-brain irradiation, radiosurgery and systemic therapy (Weil et al, 2005). Surgery or radiosurgery is an option for patients with 
one to three metastases. Whole-brain irradiation, while offering activity also in patients with $>3$ metastases, causes long-term sides effects such as memory loss and cognitive impairment. Effect of systemic therapy is limited by the blood-brain barrier. Thus, limited therapy options for symptomatic BM substantiates the urgent need for better understanding of risk factors and possibilities of prevention.

Importantly, treatment with lapatinib resulted in a decreased incidence of BM in HER-2-positive disease (Geyer et al, 2006). Other preventive measures such as prophylactic cranial radiotherapy, while well established in small-cell-lung cancer, is not routinely used in breast cancer, as no survival benefit was observed so far (Saip et al, 2009). Even screening for BM is not a part of routine follow-up, as no evidence for a benefit from early detection exists (Niwinska et al, 2007). This, however, might be rather due to the lack of appropriate selection criteria for a potential screening cohort. Therefore, a more precise definition of patients and breast cancer subtypes at high risk for early development of BM is needed (Heitz et al, 2009).

The objective of this study therefore was to determine clinical and histopathological risk factors associated with early development of BM. This might identify a high-risk population deriving the largest benefit from screening and prevention.

\section{PATIENTS AND METHODS}

Two Austrian centres contributed information relating to demographics, case history and survival. Data were processed at the Medical University of Vienna, Austria. This retrospective analysis was conducted in accordance with the ethical regulations of the Medical University of Vienna and approval by the local ethics committee was obtained.

\section{Patients}

Patients treated for symptomatic BM from breast cancer between 1996 and 2010 were identified from a breast cancer database. No routine screening for $\mathrm{BM}$ was conducted, and none of the patients available for this analysis participated in trials of BM screening or prevention. Data were analysed as of August 2011.

\section{Hormone-receptor and HER-2 status}

Oestrogen-receptor and progesterone-receptor status was assessed by immunohistochemistry (ER $\alpha$ antibody, clone 1D5, Dako A/S, Glostrup, Denmark; and PR antibody, Dako A/S). Receptor expression was estimated as the percentage of positively stained tumour cells. Results were given as $1+, 2+$ and $3+$ positive or negative staining, with a cutoff value of $<10 \%$ positive tumour cells (Hammond et al, 2010). HER-2 status was assessed by immunohistochemistry (Herceptest; Dako A/S) or dual colour fluorescent in situ hybridisation (FISH; PathVision HER-2 DNA probe kit, Vysis Inc., Downers Grove, IL, USA). Tumours were classified as HER-2-positive if they had a staining intensity of $3+$ on the Herceptest; if a score of $2+$ was gained, tumours were reanalysed by FISH (Wolff et al, 2007).

\section{Breast cancer subtypes}

Breast cancer subtypes were defined according to the results of the immunohistochemical analysis. Tumours heralding hormonereceptor expression in the absence of HER-2-receptor overexpression were summarised as belonging to the luminal subtype, without further differentiation. The HER-2 subtype was defined by overexpression of the HER-2 receptor and/or amplification of the HER-2/neu gene. Tumours were defined as triple-negative in the absence of ER, PgR as well as HER-2 expression (Anders et al, 2011; Duan et al, 2011).

\section{Treatment plan and patient evaluation}

In metastatic patients, routine re-evaluation of patients' tumour status was performed every 3 months with contrast-enhanced CT scans of the chest and the abdomen, with additional work up if indicated. In patients with early breast cancer, follow-up was done according to local protocol. Brain imaging was performed only when symptoms of CNS metastases or carcinomatous meningitis occurred. Brain metastases were diagnosed by CT and/or MRI and histologically confirmed in case neurosurgery was performed. Carcinomatous meningitis was defined as enhancement of the meninges as detected by MRI and/or detection of tumour cells in the cerebrospinal fluid. Metastatic breast cancer and BM were treated according to the current evidence-based standard of care including surgery, radiotherapy, systemic therapy, targeted therapy and endocrine treatment (Beslija et al, 2007). Follow-up of $\mathrm{BM}$ was conducted every 3 months with either contrastenhanced cranial CT or MRI scans.

\section{Study end points}

We defined brain metastases free survival (BMFS) as the interval from diagnosis of metastatic disease until the development of BM. Therefore, patients with $\mathrm{BM}$ as first site of metastatic disease were excluded from analysis of BMFS. Furthermore, we analysed the association of breast cancer subtypes with brain as first site of disease progression, number of $\mathrm{BM}$, time to development of $\mathrm{BM}$ ( $<24$ months $v s>48$ months), and development of carcinomatous meningitis.

\section{Statistical analysis}

Brain metastases free survival was estimated by the Kaplan-Meier product limit method. To test the differences between BMFS curves, the log-rank test was used. For correlation of two parameters, the $\chi^{2}$-test and the likelihood ratio were used. Twotailed $P$-values $<0.05$ were considered to indicate statistical significance. Variables exhibiting significance $(P<0.05)$ or near significance $(P<0.09)$ at univariate analysis were included into a Cox proportional hazards models.

The association of the following variables with BMFS were investigated using univariate analysis: breast cancer subtype (luminal $v s$ triple-negative $v s$ Her-2-positive), presence of pulmonary metastases, presence of any visceral metastases, age at primary diagnosis ( $>65$ years; $<35$ years), grading (grades 1 and 2 vs 3), stage at primary diagnosis (localised $v s$ metastatic) and time to progression after first diagnosis of early breast cancer $(<24$ months $v s>24$ months). Correlation analysis was performed for subtype and BM as first site of recurrence, time to progression to the brain ( $<24$ months, $>48$ months), number of $\mathrm{BM}(1-3 v s>3 \mathrm{BM})$ and presence of carcinomatous meningitis.

All statistics were calculated using statistical package for the social sciences (SPSS) 17.0 software (SPSS Inc., Chicago, IL, USA).

\section{RESULTS}

\section{Patient characteristics}

Overall, 250 patients with BM from breast cancer were identified from two Austrian centres between 1996 and 2010 (absolute incidence of breast cancer in Austria 1996-2010: 68661 patients). Thirty-seven patients had to be excluded due to incomplete information about breast cancer subtype (e.g., missing data concerning Her-2 status, hormone-receptor status). Therefore, 213 patients were available for this retrospective analysis.

According to the immunohistochemical analysis of the primary tumour, patients were divided into three groups: luminal subtype, HER-2 subtype and triple-negative subtype. Forty-six patients 
Table I Patient characteristics (a) without BM and (b) with BM as first site of progression

(a)

Entered

\begin{tabular}{|c|c|}
\hline & $\begin{array}{c}\text { Entered } \\
\text { patients }(n=169)\end{array}$ \\
\hline Characteristics & $n$ \\
\hline
\end{tabular}

Median age at first diagnosis (years)

Range

Age $>65$ years

Age $<35$ years

Grade 3 tumour

Invasive ductal carcinoma

Stage IV

Subtype

Luminal subtype

HER-2 subtype

Triple-negative subtype

\section{Adjuvant chemotherapy}

Adjuvant endocrine therapy

Adjuvant trastuzumab

Median time to progression (months)

Range

Visceral metastases

Brain as the first site of metastatic disease

Median metastatic sites

Range

Lung

Liver

Bones

Lymph nodes

Soft tissue

Skin

Others

Palliative chemotherapy before BM

Palliative endocrine therapy before BM

Palliative trastuzumab before BM

Palliative lapatinib before BM

Response to systemic therapy at time of BM diagnosis

CR

PR

SD

$\mathrm{PD}$

Median BM free survival (months)

Range

Median OS from first diagnosis (months)

Range

Median OS from diagnosis of metastatic disease

Range

Median OS from diagnosis of BM (months)

Range

(b)

Characteristics
Median age at first diagnosis (years)
Range
Age $>65$ years
Age $<35$ years

Grade 3 tumour

Invasive ductal carcinoma

Stage IV

Subtype

Luminal subtype

HER-2 subtype

Triple-negative subtype

50

25-82

17

17

116

135

32

36

102
31

115

115
53

12

121
0

0

$0-166$

2

$1-5$

47.0

42.3

48.2

28.6

32. 1

10.1

6.6

89.9

37.5

50.3

58.5

$3-218$

33

$2-125$

5.5

$0-81$

Entered patients $(n=44)$

n $\%$

54

54
$27-79$

$\begin{array}{rr}5 & 11.4 \\ 4 & 9.1 \\ 31 & 75.6 \\ 30 & 73.2 \\ 4 & 9.1 \\ & \\ 10 & 22.7 \\ 22 & 50.0 \\ 12 & 27.3\end{array}$

Table I (Continued)

(b)

Entered

patients $(n=44)$

\section{Characteristics}

Adjuvant chemotherapy

Adjuvant endocrine therapy

Adjuvant trastuzumab

median time to progression (months)

Range

Visceral metastases

Brain as only site of metastatic disease

n $\%$

80.5

$13 \quad 32.5$

18.5

$0-89$

$17 \quad 38.6$

Median metastatic sites

Range

Lung

Liver

Bones

Lymph nodes

Soft tissue

Skin

Others

22

Median OS from first diagnosis (months)

Range

Median OS from diagnosis of metastatic disease (months)

Range

Median OS from diagnosis of BM (months)

1

$1-6$

Range
29

$0-121$

$0-50$

$0-50$
Abbreviations: $\mathrm{CR}=$ complete response; $\mathrm{PR}=$ partial response; $\mathrm{SD}=$ stable disease $\mathrm{PD}=$ progressive disease; $\mathrm{BM}=$ brain metastases; $\mathrm{OS}=$ overall survival. Characteristics grading, staging, subtype are from time point of first diagnosis. Characteristics metastatic sites are from time point of diagnosis of brain metastases.

(21.6\%) belonged to the luminal subtype, 124 patients $(58.2 \%)$ to the HER-2 subtype and 43 patients $(20.2 \%)$ to the triple-negative subtype. Forty-four patients $(20.7 \%)$ had BM as first site of metastatic disease and therefore were excluded from the analysis of BMFS. All patients were treated according to the current standard of treatment for breast cancer and metastatic breast cancer, respectively (Beslija et al, 2007; Goldhirsch et al, 2007). In all, $89.9 \%$ were treated with chemotherapy-based regime for metastatic disease before the diagnosis of $\mathrm{BM}$. The remaining $10.1 \%$ of patients were treated with either endocrine monotherapy or trastuzumab monotherapy. Patient characteristics are summarised in Table 1.

\section{Brain metastases free survival}

Median BMFS was 19 months (95\% CI: 15.18-22.82) in the population of 169 patients with metastatic breast cancer who did not have BM as first site of progression. Univariate analysis revealed a significant difference in median BMFS between breast cancer subtypes. In the luminal subtype, median BMFS was 34 months (95\% CI: $23.71-44.29)$ compared with 18 months (95\% CI: 14.46-21.54) in the HER-2-positive subtype $(P=0.001$, log-rank test) and 14 months (95\% CI: $11.34-16.66)$ in the triple-negative subtype ( $P=0.001$, log-rank test) (Figure 1$)$.

In patients with lung metastases, median BMFS was 17 months (95\% CI: $14.10-19.90)$ compared with 21 months (95\% CI: $15.45-$ $26.55)$ in patients with no evidence of lung metastases $(P=0.014$, log-rank test) (Figure 2). In patients with time to extracranial progression after first diagnosis of early breast cancer of $<24$ months, median BMFS was significantly shorter compared to patients with time to extracranial progression after first diagnosis over 24 months (14 vs 24 months; $P<0.001$, log-rank test). None of the other variables included into the univariate model displayed a significant influence on BMFS (Table 2). 


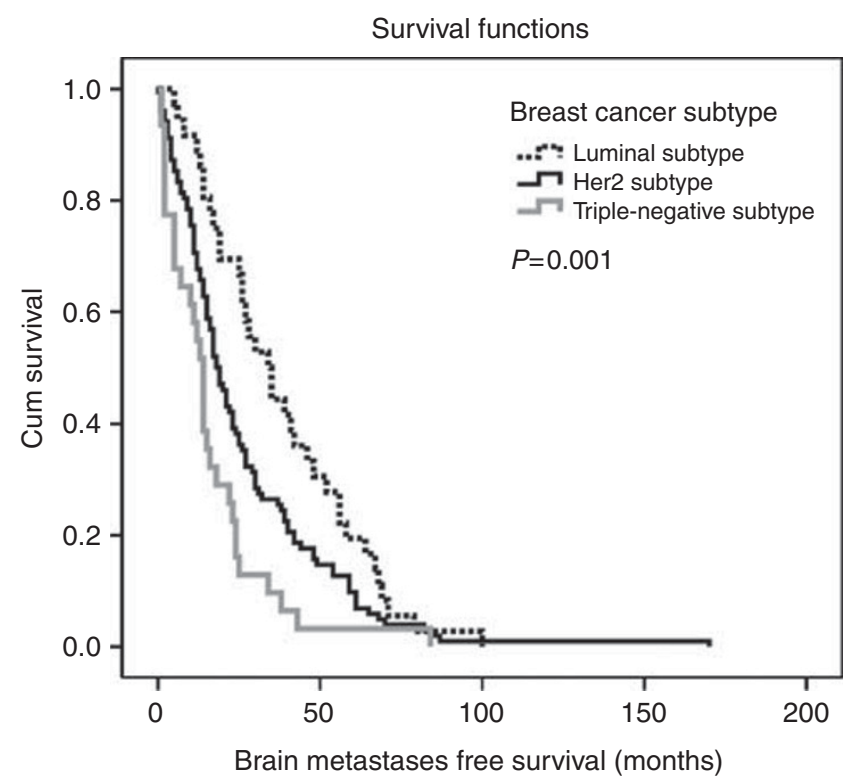

Figure I Kaplan-Meier estimates for BMFS. Median BMFS in triplenegative subtype was 14 months (95\% Cl: | | .34- |6.66) compared with 18 months (95\% Cl: | $4.46-2 \mid .54)$ in HER-2 subtype and 34 months $(95 \% \mathrm{Cl}$ : $23.7 \mathrm{I}-44.29)$ in luminal subtype $(P=0.00 \mathrm{I}$, log-rank test).

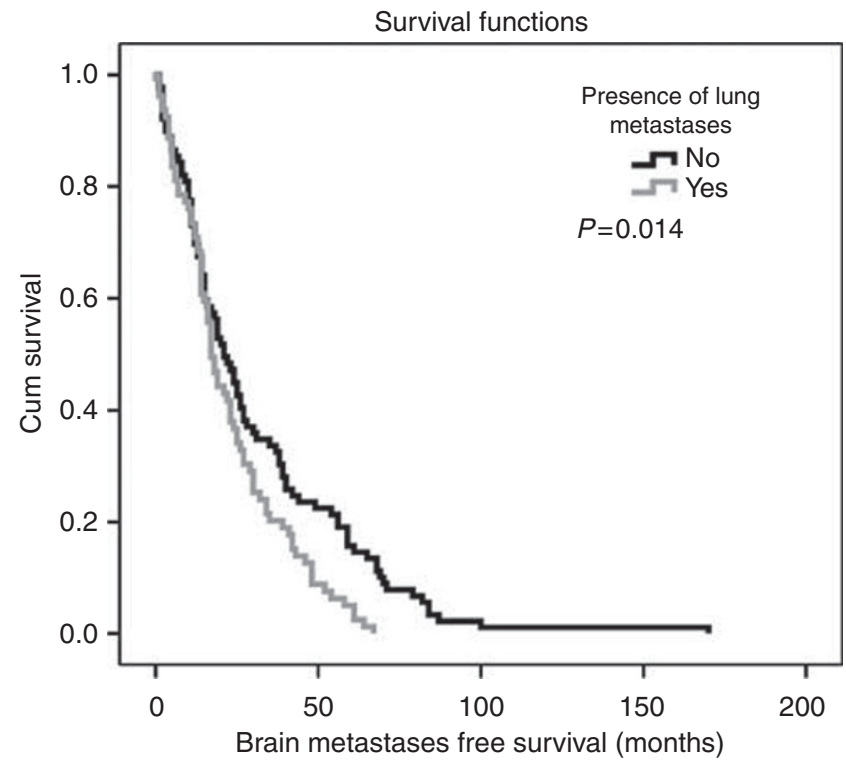

Figure 2 Kaplan-Meier estimates for BMFS. Median BMFS in patients with the presence of lung metastases was 17 months $(95 \% \mathrm{Cl}$ : 14.10 19.90) compared with 21 months (95\% Cl: $15.45-26.55)$ in patients with no evidence of lung metastases $(P=0.014$, log-rank test).

In the multivariate analysis of BMFS, presence of lung metastases and breast cancer subtype as well as time to extracranial progression after first diagnosis of early breast cancer retained statistical significance. Hazard ratio (HR) for non-luminal breast cancer subtypes was 1.51 (95\% CI: $1.17-1.95 ; P=0.002$, Cox proportional hazards model), 1.39 (95\% CI: $1.01-1.93 ; P=0.047$, Cox proportional hazards model) for presence of lung metastases and 1.49 (95\% CI: $1.07-2.08 ; P=0.019$, Cox proportional hazards model) for time to progression after first diagnosis of early breast cancer of $<24$ months, respectively.
Table 2 Univariate analysis: factors associated with brain metastases free survival (BMFS)

\begin{tabular}{|c|c|c|c|}
\hline Factor & $\begin{array}{c}\text { Median BMFS } \\
\text { (months) }\end{array}$ & $95 \% \mathrm{Cl}$ & $P$-value \\
\hline \multicolumn{4}{|l|}{ Subtype } \\
\hline Luminal subtype & 34 & $23.71-44.29$ & 0.001 \\
\hline HER-2 subtype & 18 & $|4.47-2| .54$ & \\
\hline Triple-negative subtype & 14 & $11.34-16.66$ & \\
\hline \multicolumn{4}{|l|}{ Presence of metastases } \\
\hline Visceral & 18 & $12.97-23.03$ & n.s. \\
\hline Pulmonary & 17 & $14.10-19.90$ & 0.014 \\
\hline \multicolumn{4}{|l|}{ Age at first diagnosis } \\
\hline$<35$ years & 16 & | $1.97-20.03$ & n.s. \\
\hline$>65$ years & 21 & $8.90-33.10$ & n.s. \\
\hline Grade 3 & 17 & | $3.70-20.30$ & n.s. \\
\hline Stage IV at primary diagnosis & 19 & $9.02-28.98$ & n.s. \\
\hline Time to progression $<24$ months & 14 & $|2.09-| 5.9 \mid$ & $<0.001$ \\
\hline
\end{tabular}

Abbreviation: $\mathrm{Cl}=$ confidence interval.

\section{$\chi^{2}$-test and likelihood ratio}

The likelihood ratio of developing BM as first site of metastatic disease did not differ significantly between the breast cancer subtypes (luminal subtype $21.7 \%$; HER-2 subtype $17.7 \%$; triplenegative subtype $20.7 \% ; P=0.372, \chi^{2}$-test).

On the other hand, the likelihood of being diagnosed with BM in $<24$ months (BMFS $<24$ months) correlated significantly with the breast cancer subtype. Within the luminal subtype, $30.6 \%$ (11 patients) of patients developed BM in <24 months; in the HER-2 subtype, $59.4 \%$ (60 patients) and in the triple-negative subtype, $77.4 \%$ (24 patients) of patients had a BMFS of $<24$ months $\left(P<0.001, \chi^{2}\right.$-test $)$. Furthermore, the likelihood of BMFS $>48$ months again correlated significantly with the breast cancer subtype. Only one patient $(3.2 \%)$ within the triple-negative subtype had a BFMS $>48$ months, while 12 patients $(17.6 \%)$ of the HER-2 group and 12 patients $(33.3 \%)$ of the luminal group had a BMFS of $>48$ months, respectively ( $P=0.006, \chi^{2}$-test).

In all, $92(48.7 \%)$ patients had over three BM at first diagnosis of BM. Accordingly, $32.3 \%$ of patients had a single metastasis, $9.5 \%$ had two BM and $9.5 \%$ three BM. The number of BM at time of first diagnosis of BM did not differ between the subtypes. In all, 24 patients $(58.5 \%)$ within the luminal subtype had three or less metastases, corresponding numbers for the HER-2-positive and triple-negative subtypes are $50.9 \%$ and $50.0 \%$, respectively $\left(P=0.666, \chi^{2}\right.$-test $)$.

The likelihood ratio for the development of carcinomatous meningitis again significantly correlated with breast cancer subtype. In all, $19.6 \%$ (nine patients) of the luminal subtype compared with $3.2 \%$ (four patients) of the HER-2 subtype and $9.3 \%$ (four patients) of the triple-negative subtype developed carcinomatous meningitis $\left(P=0.002, \chi^{2}\right.$-test $)$.

\section{BMFS in subsets of the HER-2-positive subtype}

In HER-2-positive patients, we further analysed whether HER-2/ER co-positivity or trastuzumab-based therapy had any influence on BMFS. In patients who received trastuzumab-based therapy before the development of BM, median BMFS was 17 months (95\% CI: 13.41-20.53) compared with 21 months (95\% CI: 8.53-33.47) in HER-2-positive patients who had not received trastuzumab-based treatment $(P=0.939$, log-rank test). Therefore, trastuzumab did not prolong BMFS.

In patients with ER/HER-2 co-positive tumours, median BMFS was 26 months (95\% CI: $16.40-35.60)$ and therefore significantly 


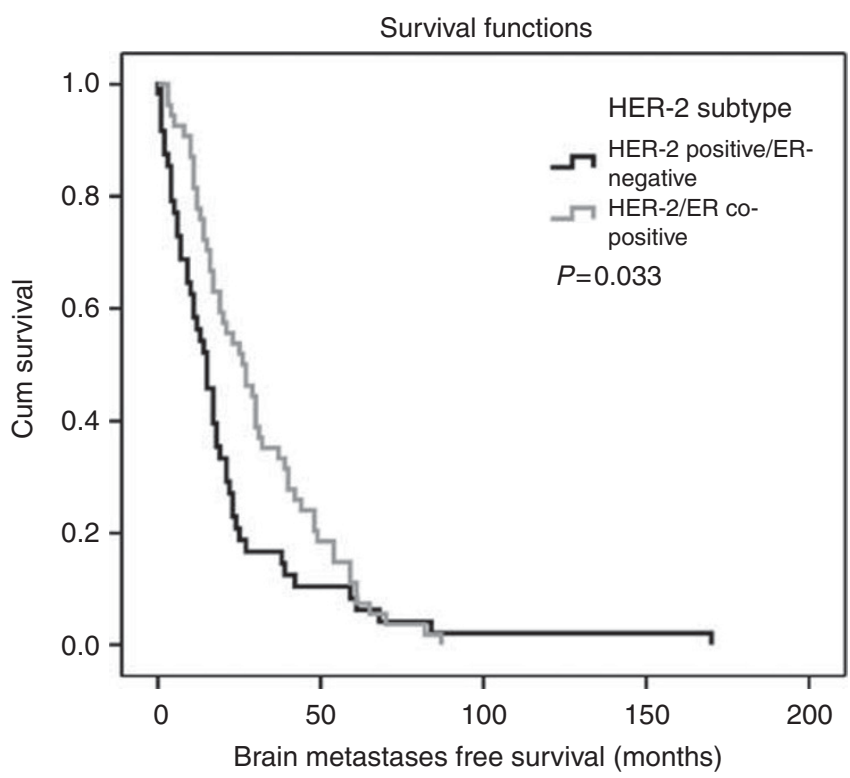

Figure 3 Kaplan-Meier estimates for BMFS. Median BMFS in HER-2/ER co-positive patients was 26 months (95\% Cl: $16.40-35.60)$ compared to (15 months; 95\% Cl: $10.77-19-23$ ) in patients with HER-2-positive/ERnegative disease $(P=0.033$, log-rank test).

longer than in patients with ER-negative/HER-2-positive disease (15 months; 95\% CI: 10.77-19-23; $P=0.033$, log-rank test) (Figure 3).

In a further step, we investigated whether palliative endocrine therapy in ER/HER-2 co-positive patients had a significant impact on BMFS, as tamoxifen has the ability to pass the blood-brain barrier. Indeed, BMFS in patients who received palliative endocrine therapy was 30 months (95\% CI: 16.17-43.83) compared with 14 months (95\% CI: 10.31 - 17.68) months in patients with ER/ HER-2 co-positive disease who did not receive prior palliative endocrine therapy $(P=0.004$, log-rank test).

\section{BMFS in subsets of the luminal subtype}

Expression of progesterone receptor did not significantly influence BMFS in patients with breast cancer of the luminal subtype. In PgR-positive patients, median BMFS was 35 months (95\% CI: $17.39-52.62)$ compared with 34 months (95\% CI: $18.35-49.66)$ in PgR-negative patients $(P=0.692$, log-rank test).

\section{Overall survival after diagnosis of BM}

Median overall survival after the diagnosis of BM was 5 months (95\% CI: 2.64-7. 36) in the luminal group, 7 months $(95 \% \mathrm{CI}$ : 4.31-969) in HER-2-positive group and 5 months (95\% CI: $1.83-$ $8.17)$ in triple-negative breast cancer patients $(P=0.364$, log-rank test). HER-2-positive patients treated with trastuzumab-based therapy after completion of local therapy for BM (surgery, radiotherapy) had a significant longer overall survival after diagnosis of BM (4 vs 14 months; 95\% CI: 2.40-5.61 vs 7.22-20.78; $P<0.001$, log-rank test).

\section{DISCUSSION}

Brain metastases are an increasing issue in modern breast cancer therapy, as up to $15 \%$ of patients with stage IV disease will eventually be diagnosed with symptomatic BM (Weil et al, 2005). Therefore, development of adequate preventive strategies is urgently required.
In the field of BM prevention in Her-2-positive disease, promising results of lapatinib were reported, a dual tyrosinekinase inhibitor of EGFR and HER-2 (Cameron et al, 2008). Other preventive strategies such as prophylactic cranial irradiation currently have no role in breast cancer treatment, as supporting data are missing (Saip et al, 2009). Also, screening for BM is not established, since early detection of BM was not found to influence survival henceforth (Niwinska et al, 2010). This, however, might result from the inclusion of patients at relatively low risk for developing BM into the respective clinical trials; therefore, a better definition of risk groups is warranted as first step to establish effective strategies of screening and prevention.

Clinical and translational research redefined breast cancer as a heterogeneous disease, divided into different subtypes defined by divergent gene expression profiles. In daily clinical practice, grading as well as immunohistochemical assessment of hormonereceptor status, Her-2, and $\mathrm{Ki}-67$ are usually used as approximation. Therefore, breast cancer is assigned to the luminal, the HER-2 or the triple-negative phenotype at first diagnosis. This classification influences estimation of prognosis and treatment decisions (Perou et al, 2000; Sorlie et al, 2001, 2003). In the present study, we show that different breast cancer subtypes associate with time to development of BM. Patients with triple-negative disease had a significantly shorter BMFS (14 months) compared with 34 months in patients with luminal tumours $(P=0.001)$. Previously, the triple-negative subtype was identified to have a higher overall risk of developing BM; furthermore, $\mathrm{BM}$ are diagnosed relatively early during the course of disease (Pestalozzi et al, 2006; Heitz et al, 2009). Here, we could demonstrate tremendous differences of BMFS in triple-negative disease in comparison to luminal tumours, as BMFS of luminal subtypes is almost doubled. This finding indicates that triple-negative breast cancer warrants further research of BM-preventive strategies (Pestalozzi, 2009).

A higher incidence of BM was observed in HER-2-positive disease as well. Different authors suggested a connection to trastuzumab, a monoclonal antibody targeting the extracellular domain of HER-2. As trastuzumab cannot penetrate the bloodbrain barrier, the CNS becomes a safe haven for tumour cells (Clayton et al, 2004). Also, improved control of systemic disease may eventually lead to the 'unmasking' of BM (Lin and Winer, 2007). In our analysis, BMFS within the HER-2 subtype was 18 months and was significantly different from the other two subtypes $(P=0.001)$. Compared with luminal cancers, shorter BMFS was observed in Her-2-positive disease, while BMFS was longer compared with triple-negative tumours. No influence of trastuzumab-based therapy on BMFS was observed. This finding indicates that biological behaviour rather than systemic treatment defines the risk for early or late development of BM in patients with HER2-positive breast cancer (Burstein et al, 2005; Pestalozzi et al, 2006; Lin and Winer, 2007).

Several studies postulated the absence of ER expression as an unfavourable factor for the probability of developing BM (Slimane et al, 2004; Weil et al, 2005). Therefore, we performed an analysis of BMFS in the HER-2-positive subtype in dependence of ER expression. Patients with ER/HER-2 co-positive disease were shown to have significantly longer BMFS compared with patients with ER-negative/HER-2-positive disease (26 months vs 15 months; $P=0.033$ ). This once again shows that the Her-2-positive phenotype comprises heterogeneous subtypes.

Brain metastases are usually diagnosed rather late in the course of metastatic disease (Weil et al, 2005). Previous studies indicate a correlation of visceral and pulmonary metastases and the occurrence of BM (Weil et al, 2005; Kennecke et al, 2010). Our findings further support this investigation, as pulmonary metastases remained a significant risk factor associated with shorter BMFS in the Cox regression model (HR 1.49; $P=0.016$ ). Therefore, we suggest that patients with triple-negative tumours and pulmonary metastases might be the most suitable 
group for prospective trials investigating strategies of screening and prevention.

The number of $\mathrm{BM}$ is an important factor for prognosis as well as treatment, as surgery or radiosurgery is usually only applied in patients with oligometastatic (1-3 metastases) disease (Kamar and Posner, 2010; Niwinska et al, 2011a,b). Recently, an influence of breast cancer subtypes on the number of BM at first diagnosis was postulated. Oestrogen-receptor-positive patients, according to one study, might be more likely to develop oligometastatic brain involvement (Garg et al, 2011). In our homogenous, large collective, however, we cannot support those findings; the likelihood for oligometastatic involvement did not differ between the breast cancer subtypes.

Carcinomatous meningitis, just like BM, occurs late during the course of the disease and treatment options are very limited (de Azevedo et al, 2011). While breast cancer subtype influences overall survival after the diagnosis of carcinomatous meningitis, little is known about risk factors (Lee et al, 2011; Niwinska et al, 2011a,b). In our study, patients with luminal subtype were at

\section{REFERENCES}

Anders CK, Deal AM, Miller CR, Khorram C, Meng H, Burrows E, Livasy C, Fritchie K, Ewend MG, Perou CM, Carey LA (2011) The prognostic contribution of clinical breast cancer subtype, age, and race among patients with breast cancer brain metastases. Cancer 117(8): $1602-1611$

Beslija S, Bonneterre J, Burstein H, Cocquyt V, Gnant M, Goodwin P, Heinemann V, Jassem J, Kostler WJ, Krainer M, Menard S, Petit T, Petruzelka L, Possinger K, Schmid P, Stadtmauer E, Stockler M, Van Belle S, Vogel C, Wilcken N, Wiltschke C, Zielinski CC, Zwierzina H (2007) Second consensus on medical treatment of metastatic breast cancer. Ann Oncol 18(2): 215-225

Burstein HJ, Lieberman G, Slamon DJ, Winer EP, Klein P (2005) Isolated central nervous system metastases in patients with HER2-overexpressing advanced breast cancer treated with first-line trastuzumab-based therapy. Ann Oncol 16(11): $1772-1777$

Cameron D, Casey M, Press M, Lindquist D, Pienkowski T, Romieu CG, Chan S, Jagiello-Cruszfeld A, Kaufman B, Crown J, Chan A, Campone M, Viens P, Davidson N, Gorbounova V, Raats JI, Skarlos D, Newstat B, Roychowdhury D, Paoletti P, Oliva C, Rubin S, Stein S, Geyer CE (2008) A phase III randomized comparison of lapatinib plus capecitabine versus capecitabine alone in women with advanced breast cancer that has progressed on trastuzumab: updated efficacy and biomarker analyses. Breast Cancer Res Treat 112(3): 533-543

Clayton AJ, Danson S, Jolly S, Ryder WD, Burt PA, Stewart AL, Wilkinson PM, Welch RS, Magee B, Wilson G, Howell A, Wardley AM (2004) Incidence of cerebral metastases in patients treated with trastuzumab for metastatic breast cancer. Br I Cancer 91(4): 639-643

de Azevedo CR, Cruz MR, Chinen LT, Peres SV, Peterlevitz MA, de Azevedo Pereira AE, Fanelli MF, Gimenes DL (2011) Meningeal carcinomatosis in breast cancer: prognostic factors and outcome. J Neurooncol 104(2): $565-572$

Duan XF, Dong NN, Zhang T, Li Q (2011) The prognostic analysis of clinical breast cancer subtypes among patients with liver metastases from breast cancer. Int J Clin Oncol; e-pub ahead of print 27 October 2011; doi:10.1007/s10147-011-0336-x

Garg RJ, Marsh JC, Rao RD, Griem SKL (2011) Predictive factors for oligometastatic $v s$ non-oligometastatic involvement of the central nervous system by brain metastases from breast cancer. 2011 Breast Cancer Symposium

Geyer CE, Forster J, Lindquist D, Chan S, Romieu CG, Pienkowski T, Jagiello-Gruszfeld A, Crown J, Chan A, Kaufman B, Skarlos D, Campone M, Davidson N, Berger M, Oliva C, Rubin SD, Stein S, Cameron D (2006) Lapatinib plus capecitabine for HER2-positive advanced breast cancer. $N$ Engl J Med 355(26): 2733-2743

Goldhirsch A, Wood WC, Gelber RD, Coates AS, Thurlimann B, Senn HJ (2007) Progress and promise: highlights of the international expert consensus on the primary therapy of early breast cancer 2007. Ann Oncol 18(7): $1133-1144$

Hammond ME, Hayes DF, Dowsett M, Allred DC, Hagerty KL, Badve S, Fitzgibbons PL, Francis G, Goldstein NS, Hayes M, Hicks DG, Lester S, higher risk for the development of carcinomatous meningitis compared to patients with HER-2 or triple-negative disease $(19.6 \%$ vs $3.2 \%$ vs $9.3 \% ; P=0.002)$. Although the small sample size has to be taken into account, this apparent contradiction to solid BM warrants further investigation.

In conclusion, our study shows that patients with triple-negative as well as patients with ER-negative/HER-2-positive disease are at highest risk for developing BM early during their course of disease. The risk is further raised by the presence of pulmonary metastases. This analysis might help in defining the optimal breast cancer patient population for future prospective trials of BM screening and prevention.

\section{ACKNOWLEDGEMENTS}

Apart from the authors, the following persons contributed to this study: Sabine Fromm, Gabriela Altorjai, Gudrun Boeckmann, Alexander DeVries and Carina Dinhof.
Love R, Mangu PB, McShane L, Miller K, Osborne CK, Paik S, Perlmutter J, Rhodes A, Sasano H, Schwartz JN, Sweep FC, Taube S, Torlakovic EE, Valenstein P, Viale G, Visscher D, Wheeler T, Williams RB, Wittliff JL, Wolff AC (2010) American Society of Clinical Oncology/College Of American Pathologists guideline recommendations for immunohistochemical testing of estrogen and progesterone receptors in breast cancer. $J$ Clin Oncol 28(16): 2784-2795

Heitz F, Harter P, Lueck HJ, Fissler-Eckhoff A, Lorenz-Salehi F, ScheilBertram S, Traut A, du Bois A (2009) Triple-negative and HER2overexpressing breast cancers exhibit an elevated risk and an earlier occurrence of cerebral metastases. Eur J Cancer 45(16): 2792-2798

Kamar FG, Posner JB (2010) Brain metastases. Semin Neurol 30(3): $217-235$

Kennecke H, Yerushalmi R, Woods R, Cheang MC, Voduc D, Speers CH, Nielsen TO, Gelmon K (2010) Metastatic behavior of breast cancer subtypes. J Clin Oncol 28(20): 3271-3277

Kiely BE, Soon YY, Tattersall MH, Stockler MR (2011) How long have I got? Estimating typical, best-case, and worst-case scenarios for patients starting first-line chemotherapy for metastatic breast cancer: a systematic review of recent randomized trials. J Clin Oncol 29(4): 456- 463

Lee S, Ahn HK, Park YH, Nam do H, Lee JI, Park W, Choi DH, Huh SJ, Park KT, Ahn JS, Im YH (2011) Leptomeningeal metastases from breast cancer: intrinsic subtypes may affect unique clinical manifestations. Breast Cancer Res Treat 129(3): 809-817

Lin NU, Winer EP (2007) Brain metastases: the HER2 paradigm. Clin Cancer Res 13(6): $1648-1655$

Niwinska A, Pogoda K, Murawska M, Niwinski P (2011a) Factors influencing survival in patients with breast cancer and single or solitary brain metastasis. Eur J Surg Oncol 37(7): 635-642

Niwinska A, Tacikowska M, Murawska M (2010) The effect of early detection of occult brain metastases in HER2-positive breast cancer patients on survival and cause of death. Int J Radiat Oncol Biol Phys 77(4): $1134-1139$

Niwinska A, Tacikowska M, Pienkowski T (2007) Occult brain metastases in HER2-positive breast cancer patients: frequency and response to radiotherapy. Acta Oncol 46(7): 1027-1029

Niwinska AM, Rudnicka H, Murawska M (2011b) Breast cancer carcinomatous meningitis: differences in survival depending on biological subtype, performance status, and treatment methods. J Clin Oncol 29: suppl abstr 1073

Perou CM, Sorlie T, Eisen MB, van de Rijn M, Jeffrey SS, Rees CA, Pollack JR, Ross DT, Johnsen H, Akslen LA, Fluge O, Pergamenschikov A, Williams C, Zhu SX, Lonning PE, Borresen-Dale AL, Brown PO, Botstein D (2000) Molecular portraits of human breast tumours. Nature 406(6797): $747-752$

Pestalozzi BC (2009) Brain metastases and subtypes of breast cancer. Ann Oncol 20(5): $803-805$

Pestalozzi BC, Zahrieh D, Price KN, Holmberg SB, Lindtner J, Collins J, Crivellari D, Fey MF, Murray E, Pagani O, Simoncini E, CastiglioneGertsch M, Gelber RD, Coates AS, Goldhirsch A (2006) Identifying breast 
cancer patients at risk for Central Nervous System (CNS) metastases in trials of the International Breast Cancer Study Group (IBCSG). Ann Oncol 17(6): 935-944

Saip P, Cicin I, Eralp Y, Karagol H, Kucucuk S, Cosar Alas R, Yavuz E, Dincer M, Saglam E, Topuz E (2009) Identification of patients who may benefit from the prophylactic cranial radiotherapy among breast cancer patients with brain metastasis. J Neurooncol 93(2): 243-251

Sanna G, Franceschelli L, Rotmensz N, Botteri E, Adamoli L, Marenghi C, Munzone E, Cossu Rocca M, Verri E, Minchella I, Medici M, Catania C Magni E, Goldhirsch A, Nole F (2007) Brain metastases in patients with advanced breast cancer. Anticancer Res 27(4C): 2865-2869

Slimane K, Andre F, Delaloge S, Dunant A, Perez A, Grenier J, Massard C, Spielmann M (2004) Risk factors for brain relapse in patients with metastatic breast cancer. Ann Oncol 15(11): 1640-1644

Sorlie T, Perou CM, Tibshirani R, Aas T, Geisler S, Johnsen H, Hastie T, Eisen MB, van de Rijn M, Jeffrey SS, Thorsen T, Quist H, Matese JC, Brown PO, Botstein D, Eystein Lonning P, Borresen-Dale AL (2001) Gene expression patterns of breast carcinomas distinguish tumor subclasses with clinical implications. Proc Natl Acad Sci USA 98(19): 10869-10874 Sorlie T, Tibshirani R, Parker J, Hastie T, Marron JS, Nobel A, Deng S, Johnsen H, Pesich R, Geisler S, Demeter J, Perou CM, Lonning PE, Brown PO, Borresen-Dale AL, Botstein D (2003) Repeated observation of breast tumor subtypes in independent gene expression data sets. Proc Natl Acad Sci USA 100(14): 8418-8423

Weil RJ, Palmieri DC, Bronder JL, Stark AM, Steeg PS (2005) Breast cancer metastasis to the central nervous system. Am J Pathol 167(4): $913-920$

Wolff AC, Hammond ME, Schwartz JN, Hagerty KL, Allred DC, Cote RJ, Dowsett M, Fitzgibbons PL, Hanna WM, Langer A, McShane LM, Paik S, Pegram MD, Perez EA, Press MF, Rhodes A, Sturgeon C, Taube SE, Tubbs R, Vance GH, van de Vijver M, Wheeler TM, Hayes DF (2007) American Society of Clinical Oncology/College of American Pathologists guideline recommendations for human epidermal growth factor receptor 2 testing in breast cancer. J Clin Oncol 25(1): 118-145

This work is published under the standard license to publish agreement. After 12 months the work will become freely available and the license terms will switch to a Creative Commons Attribution-NonCommercial-Share Alike 3.0 Unported License. 